\title{
Reflex modification during habituation of a startle response
}

\author{
HOWARD S. HOFFMAN, MICHELLE E. COHEN, and CHRISTINE CORSO \\ Bryn Mawr College, Bryn Mawr, Pennsylvania
}

\begin{abstract}
Reflex modification describes the phenomenon in which an elicited reflex is inhibited when a weak stimulus is presented approximately $100 \mathrm{msec}$ prior to the reflex-eliciting event. During habituation procedures, rats were repeatedly exposed to a startle-eliciting noise either with or (in a separate session) without a preceding reflex-inhibiting stimulus and were then tested with the same or the contrasting condition. When tested with a reflex-eliciting stimulus presented alone, rats exposed to reflex modification during habituation procedures exhibited responses of the same amplitude as when they had been exposed to the startle-eliciting stimulus alone from the beginning. Moreover, when contrasting conditions were employed, contrasting results were obtained. The finding that reflex modification and habituation have largely independent effects implies that these effects are probably mediated by separate neural systems.
\end{abstract}

When a relatively weak stimulus such as a mild tone precedes an intense (reflex-eliciting) stimulus by an appropriate interval (approximately $100 \mathrm{msec}$ ), the amplitude of the elicited reaction is often reduced. The effect is called reflex modification. A number of investigations (see Hoffman \& Ison, 1980, for review) have revealed that when the intensity of the reflex-eliciting stimulus is systematically varied, the magnitude of the inhibitory effect of a given reflex-modifying signal (e.g., the amount of reflex modification) is independent of the amplitude of the overt reflex as determined by the intensity of the reflex-eliciting stimulus. Thus, a given reflex-modifying tone inhibits the large reaction to an intense reflex-eliciting stimulus by the same amount that it inhibits the smaller reaction evoked by a more moderate signal. As noted by Hoffman and Ison, this pattern of results indicates that reflex modification and reflex elicitation are largely independent processes and that they are mediated by separate neural systems, an implication that is supported by the results of recent neurophysiological investigations (Leitner, Powers, \& Hoffman, 1980; Leitner, Powers, Stitt, \& Hoffman, 1981). The research reported here sought to determine how habituation of the rat's startle reflex to an intense acoustic signal proceeds when a mild reflex-modifying tone is employed to inhibit the overt reaction throughout the course of the habituation procedure.

At the outset, it seemed possible that these operations might yield any of a variety of results. For example, by inhibiting the overt reaction, one might prevent or perhaps retard the habituation process so that, when subsequently confronted by the reflex-eliciting signal presented alone

This research was supported by National Institute of Health Grant HD 10511. Requests for reprints should be sent to Howard S. Hoffman, Department of Psychology, Bryn Mawr College, Bryn Mawr, PA 19010. (i.e., with no prior reflex-modifying tone), a rat would show less habituation (i.e., larger reflexes) than when it had experienced the same sequence of reflex-eliciting stimuli presented alone. A finding of this sort would point to the conclusion that reflex modification and habituation procedures interact to reduce the effects of the latter. Another possible outcome was that, by inhibiting the elicited reflexes, one might somehow facilitate the habituation process so that animals would exhibit smaller (i.e., more fully habituated) reflexes when subsequently confronted by the reflex-eliciting stimulus presented alone than when the response had been inhibited during habituation procedures. Such a finding would also suggest that reflex-modification and habituation procedures interact but that the effect is to enhance the habituation process. The third prospect was that reflex modification would prove to be independent of habituation-in which case animals whose reflexes were inhibited during habituation procedures would exhibit, during subsequent tests, reactions of the same amplitude as animals whose reflexes were not inhibited during prior habituation procedures. Should this prove to be the case, it would mean that, like reflex modification and reflex elicitation, reflex modification and habituation have largely independent effects, a result that would imply that the two effects are probably mediated by separate neural systems.

\section{METHOD}

\section{Subjects}

The subjects were 12 180-day-old male albino rats obtained from Charles River Breeding Laboratories.

\footnotetext{
Apparatus

The research was conducted in an Industrial Acoustics Corporation sound-attenuating chamber with an ambient noise level of less than $20 \mathrm{~dB}$ for frequencies above $100 \mathrm{~Hz}$. The apparatus consisted of a small test cage $(20.3 \times 15.2 \mathrm{~cm})$ constructed of stainless steel rods suspended from a rigid superstructure by four thin pieces of epoxy and fiberglass
} 
laminate. An aluminum rod with a magnet in its distal end was mounted on top of the chamber so that the magnet rode inside a coil that was fastened to the superstructure. With this arrangement, any sudden movement of the chamber, as, for example, during a startle reflex, would cause the magnet to move in the coil and generate an electrical current. This current was filtered (to eliminate stimulus artifacts) and amplified before being passed to a Coulbourn peak detector (Model S76-31), which sampled responses for $150 \mathrm{msec}$ after the onset of the startle stimuli. The output of the peak detector was digitized and strobed to a Radio Shack TRS-80 computer.

Reflex-modifying tones were produced by a Coulbourn precision signal generator (Model S81-06) and amplified by one channel of a Crown audio power amplifier (Model DC-300). The startle-eliciting stimulus was produced by a Coulbourn random noise generator (Model S81-02) and amplified by the other channel of the Crown unit. Startle-eliciting signals were presented through an Altec midrange exponential horn (Model 31A) fitted with an Altec compression driver (Model Z30C) mounted inside the sound-attenuating chamber a few inches from the test cage. Reflex-modifying tones (when used) were presented through a Realistic Piezo Super Tweeter that was mounted on the opposite side of the test cage.

\section{Procedure}

The subjects were placed in the testing cage for an adaptation period of $5 \mathrm{~min}$, during which no stimuli were presented. This procedure allowed time for initial exploratory behavior to subside. There were two basic stimulus configurations: (1) a startle-eliciting noise burst alone, and (2) a reflex-modifying tone followed $100 \mathrm{msec}$ later by a startleeliciting noise burst. The reflex-modifying tone had a frequency of $2.5 \mathrm{kHz}$, an intensity of $60 \mathrm{~dB}$ SPL, a duration of $50 \mathrm{msec}$, and a $20-$ $\mathrm{msec}$ rise/fall time. The startle-eliciting stimulus was a 110-dB SPL whitenoise burst of 20 -msec duration with a .1-msec rise/fall time. There were four experimental conditions; in each, the subjects received 200 habituation trials at an intertrial interval of $15 \mathrm{sec}$. Habituation trials were followed $15 \mathrm{sec}$ later by a sequence of 20 test trials at a 15 -sec intertrial interval. The conditions were: Condition 1-startle-eliciting signal alone during both habituation and test trials; Condition 2-startle eliciting signal alone during habituation trials followed by reflexmodification procedures during test trials; Condition 3-reflexmodification procedures during habituation trials followed by the presentation of the startle-eliciting signal alone during test trials; and Condition 4-reflex-modification procedures during both habituation and test trials.

Each subject received all four experimental conditions in random order. All subjects were tested with one condition per session, and sessions occurred on alternate days. Pilot work had suggested that, with the conditions to be used, recovery from the effects of a given habituation and test procedure was likely to be complete by the end of $48 \mathrm{~h}$.

\section{RESULTS}

Figure 1 shows the subjects' mean response amplitude over 11 blocks of 20 trials each during habituation and test procedures. The inhibited reactions followed the same course as the uninhibited startle reactions. A two-way repeated-measures ANOVA of the data from the habituation procedures yielded significant differences across trial blocks $[\mathrm{F}(9,429)=12.92, \mathrm{p}<.05]$ and significant differences between conditions $[F(3,429)=35.17$, $\mathrm{p}<.05$ ], with no significant interaction. Newman-Keuls analysis $(p<.05)$ of the conditions effect revealed that during the habituation procedure the two startle-alone conditions combined (Conditions 1 and 2) were significantly different from the two reflex-modification conditions (Conditions 3 and 4) combined, but that the small observed differences within each pair of conditions were not significant.

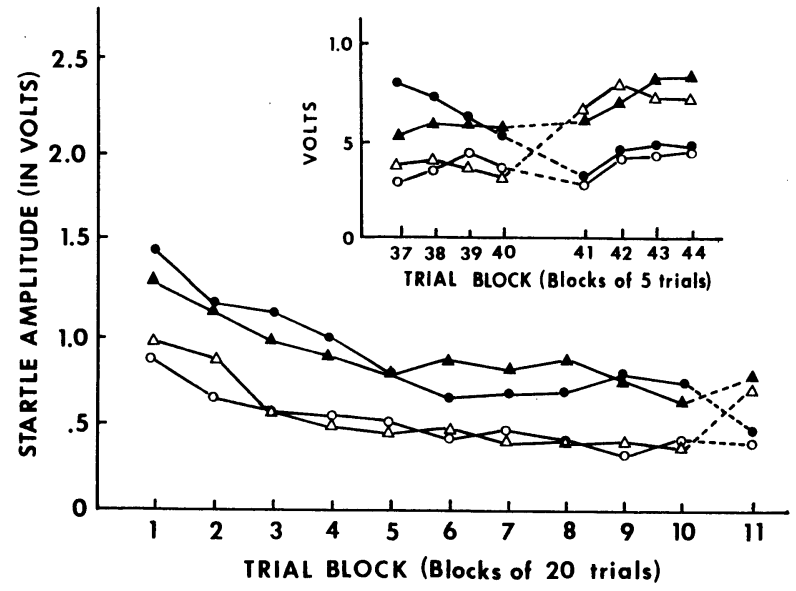

Figure 1. Mean startle amplitude (across subjects) per block of 20 trials during habituation and test procedures. Closed circles indicate responses when subjects were exposed to the startle-eliciting stimulus presented alone during habituation procedures and were exposed to the same startle-eliciting stimulus presented alone during the test sequence (Block 11). Closed triangles indicate responses when subjects were exposed to the startle-eliciting stimulus presented alone during habituation and were exposed to the same startleeliciting stimulus preceded by a reflex-modifying tone during the test sequence. Open circles and open triangles indicate the responses when subjects were exposed to contrasting conditions in which a reflex-modifying tone preceded the startle-eliciting signal during habituation procedures and the reflex-modifying tone was then either omitted (open triangles) or continued (open circles) during the test procedure. The inset shows the details of the response levels during the 4 blocks of 5 trials that together constituted the final block of 20 habituation trials (Block 10) and the block of 20 test trials (Block 11).

A two-way repeated-measures ANOVA was performed on the data from Trial Blocks 10 and 11. These data are shown in greater detail in the inset of Figure 1. The statistical analysis revealed a significant main effect for conditions $[\mathrm{F}(3,77)=4.25, \mathrm{p}<.05]$, as well as a significant block $\times$ condition interaction $[\mathrm{F}(3,77)=5.02$, $\mathrm{p}<.05]$, but there was no significant change across blocks. Results of an analysis of the simple main effects indicated that the four conditions differed significantly during the terminal habituation trial block $[F(3,77)=$ 4.06, $\mathrm{p}<.05$ ] and that they also differed significantly during the test block $[F(3,77)=5.23, p<.05]$. Subsequent Newman-Keuls analyses $(p<.05)$ revealed that within the final habituation block, both of the conditions that entailed presentation of the startle-eliciting stimulus during habituation differed significantly from each of the two conditions that entailed reflex-modification procedures during habituation, but neither of these conditions differed significantly from each other. A similar result was obtained in the analysis of the data from the test block. Within this block, the two conditions that entailed presentation of the startle-eliciting stimulus alone during the test differed significantly from each of the conditions that entailed reflex modification during the test, but neither of these conditions differed significantly from each other. Further tests of the simple main effects revealed that within Condition 2 (presentation of the startle-eliciting 
stimulus alone during habituation, reflex modification during test), the uninhibited startle reactions during the terminal habituation block were significantly larger than the inhibited startle reactions during the test block. In Condition 3 (reflex modification during habituation, presentation of the startle-eliciting stimulus alone during the test), the opposite results were obtained: The inhibited startle reactions during the terminal habituation block were significantly smaller than the uninhibited startle reactions during the test block. The other two conditions (presentation of the startle-eliciting stimulus alone during both habituation and test procedures and reflex modification during both habituation and test procedures) yielded no significant differences between the habituation and test block.

\section{DISCUSSION}

Considered together, these results indicate that there were no enduring effects of the inhibition afforded by the reflex-modification procedure; the inhibitory effects of the reflex-modifying stimulus were limited only to those trials on which the reflex-modifying stimulus was presented.
In short, habituation followed essentially the same course when responses were uninhibited and hence large and when they were inhibited and hence small.

Of the three possible outcomes considered earlier, the one that occurred here implies that reflex modification and habituation are separate and, as far as these data are concerned, independent processes. There was no evidence of interaction between reflex modification and habituation at any level of this work. The major implication of this finding is that habituation and reflex modification are probably mediated by separate neural systems.

\section{REFERENCES}

Hoffman, H. S., \& Ison, J. R. (1980). Reflex modification in the domain of startle: I. Some empirical findings and their implications for how the nervous system processes sensory input. Psychological Review, 87, 175-189.

Leitner, D. S., Powers, A. S., \& Hoffman, H. S. (1980). The neural substrate of the startle response. Physiology \& Behavior, 25, 291-297.

Leitner, D. S., Powers, A. S., Stitt, C. L., \& Hoffman, H. S. (1981). Midbrain reticular formation involvement in the inhibition of acoustic startle. Physiology \& Behavior, 26, 259-268.

(Manuscript received for publication July 2, 1984.) 